\title{
Maintaining Arrays of Contiguous Objects
}

\author{
Michael A. Bender ${ }^{1}$, Sándor P. Fekete ${ }^{2}$, Tom Kamphans ${ }^{2 \star}$, and Nils Schweer ${ }^{2}$ \\ 1 Department of Computer Science State University of New York at Stony Brook \\ Stony Brook, NY 11794-4400, USA \\ 2 Braunschweig University of Technology, Department of Computer Science, \\ Algorithms Group, 38106 Braunschweig, Germany
}

\begin{abstract}
In this paper we consider methods for dynamically storing a set of different objects ("modules") in a physical array. Each module requires one free contiguous subinterval in order to be placed. Items are inserted or removed, resulting in a fragmented layout that makes it harder to insert further modules. It is possible to relocate modules, one at a time, to another free subinterval that is contiguous and does not overlap with the current location of the module. These constraints clearly distinguish our problem from classical memory allocation. We present a number of algorithmic results, including a bound of $\Theta\left(n^{2}\right)$ on physical sorting if there is a sufficiently large free space and sum up NP-hardness results for arbitrary initial layouts. For online scenarios in which modules arrive one at a time, we present a method that requires $O(1)$ moves per insertion or deletion and amortized cost $O\left(m_{i} \lg \hat{m}\right)$ per insertion or deletion, where $m_{i}$ is the module's size, $\hat{m}$ is the size of the largest module and costs for moves are linear in the size of a module.
\end{abstract}

\section{Introduction}

Maintaining a set of objects is one of the basic problems in computer science. As even a first-year student knows, allocating memory and arranging objects (e.g., sorting or garbage collection) should not be done by moving the objects, but merely by rearranging pointers.

The situation changes when the objects to be sorted or placed cannot be rearranged in a virtual manner, but require actual physical moves; this is the case in a densely packed warehouse, truck or other depots, where items have to be added or removed. Similarly, allocating space in a fragmented array is much harder when one contiguous interval is required for each object: Even when there is sufficient overall free space, placing a single item may require rearranging the other items in order to create sufficient connected free space. This scenario occurs for the application that initiated our research: Maintaining modules on a Field Programmable Gate Array (FPGA); reconfigurable chips that consist of a two-dimensional array of processing units. Each unit can perform one basic operation depending on its configuration, which can be changed during runtime. A module is a configuration for a set of processing units wired together to fulfill

\footnotetext{
* Supported by DFG grant FE 407/8-3, project "ReCoNodes"
} 


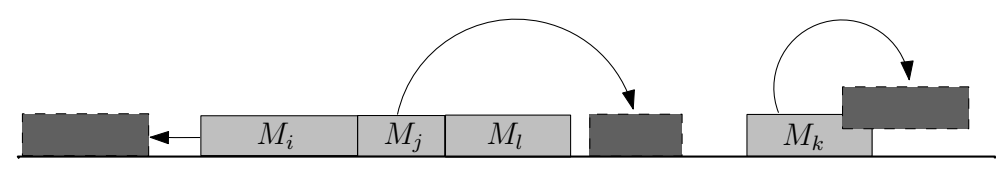

Fig. 1. A module corresponds to a set of columns on an FPGA. Each module occupies a contiguous block of array cells. Module $M_{i}$ is shifted and module $M_{j}$ is flipped. The move of module $M_{k}$ is forbidden, because the current and the target position overlap. If these kind of moves would be allowed connecting the total free space could always be done by shifting all modules to one side.

a certain task. As a lot of FPGAs allow only whole columns to be reconfigured, we allow the modules to occupy only whole columns on the FPGA (and deal with a one-dimensional problem). Moreover, because the layout of the modules (i.e., configurations and interconnections of the processing units) is fixed, we have to allocate connected free space for a module on the FPGA. In operation, different modules are loaded onto the FPGA, executed for some time and are removed when their task is fulfilled, causing fragmentation on the FPGA. When fragmentation becomes too high (i.e., we cannot place modules, although there is sufficent free space, but no sufficent amount of connected free space), the execution of new task has to be delayed until other tasks are finished and the corresponding modules are removed from the FPGA. To reduce the delay, we may reduce fragmentation by moving modules. Moving a module means to stop its operation, copy the module to an unoccupied space, restart the module in the new place, and declare the formerly occupied space of the module as free space; see Figure 1. Thus, it is important that the current and the target position of the module are not overlapping (i.e., they do not share a column). This setting gives rise to two approaches: We may either use simple placing strategies such as first fit and compact the whole FPGA when necessary (as discussed in [1]), or use more elaborated strategies that organize the free space and avoid the need for complete defragmentations.

Related Work. There is a large body of work on storage allocation; e.g., [2] for an overview and $[3,4]$ for competitive analysis of some algorithms. Many storage allocation algorithms also have analogues in bin packing [5]. The salient feature of most traditional memory-allocation and bin-packing heuristics is that once an item is allocated, it cannot be moved, unlike the model is this paper. There is also a tremendous amount of work on classical sorting (see, e.g., [6]).

Physical allocation, where elements can be placed and then moved, has received less attention. Itai, Konheim, and Rodeh consider maintaining $n$ unit-size objects sorted in an $O(n)$ sized array by appropriately maintaining a linear number of gaps interspersed between the elements at an amortized cost of $O\left(\lg ^{2} n\right)$ per insert, and the problem is deamortized in [7]. The packed memory array Bender, Demaine, and Farach-Colton [8] and Bender and $\mathrm{Hu}[9]$ investigate a similar problem in the context of external-memory and cache-oblivious algorithms. Bender, Farach-Colton, and Mosteiro [10] show that probabilistically a modified insertion sort runs in $O(n \lg n)$ by leaving appropriate gaps between 
elements. In these papers, elements have unit size and there is a fixed order that needs to be maintained dynamically, unlike the problem in this paper.

A different problem is described by [11], who consider densely packed physical storage systems for the U.S. Navy, based on the classical 15-puzzle, where items can be moved to an adjacent empty cell. How should one arrange and maintain the set of free cells, and how can objects be retrieved as quickly as possible?

Finally, if the sequence of modules (i.e., their size, processing time, and arrival time) is fully known, then the problem can be stated as a strip packing problem (without rotation) with release times for rectangles with widths and heights corresponding to the module's size and time, respectively. There is a $(1+\varepsilon)$ approximation for (classical) offline strip packing [12]. For the case with release times, Augustine et al. [13] give a $O(\lg n)$ approximation and a 3 -approximation for heights bounded by one. For approaches from the FPGA community see [1] and the references cited in this paper.

This Paper. Dealing with arrangements of physical objects or data that require contiguous memory allocation and nonoverlapping moves gives rise to a variety of problems that are quite different from virtual storage management:

- Starting configuration vs. full management. We may be forced to start from an arbitrary configuration, or be able to control the placement of objects.

- Physical sorting. Even when we know that it is possible to achieve connected free space, we may not want to get an arbitrary arrangement of objects, but may be asked to achieve one in which the objects are sorted by size.

- Low-cost insertion. We may be interested in requiring only a small number of moves per insertion, either on average, or in the worst case.

- Objective functions. Depending on the application scenario, the important aspects may differ: We may want to minimize the moves for relocating objects, or the total mass that is moved. Alternatively, we may perform only very few moves (or none at all), at the expense of causing waiting time for the objects that cannot be placed; this can be modeled as minimizing the makespan of the corresponding schedule.

Main Results. Our main results are as follows:

- We demonstrate that sorting the modules by size may require $\Omega\left(n^{2}\right)$ moves.

- We show that keeping the modules in sorted order is sufficient to maintain connected free space and to achieve an optimal makespan, requiring $O(n)$ moves per insertion or deletion.

- We give an alternative strategy that guarantees connected free space; in most steps, this requires $O(1)$ moves for insertion, but may be forced to switch to sorted order in $O\left(n^{2}\right)$ moves for high densities.

- We present an online method that needs $O(1)$ moves per insertion or deletion.

- We perform a number of experiments to compare the strategies.

- For the sake of completeness, we briefly cite and sketch that it is strongly NP-hard to find an optimal defragmentation sequence when we are forced to start with an arbitrary initial configuration, that (unless $\mathrm{P}$ is equal to NP) it is impossible to approximate the maximal achievable free space within any constant, and prove that achieving connected space is always possible for low module density. 
The rest of this paper is organized as follows. In Section 2, we introduce the problem and notation. Section 3 discusses aspects of complexity for a (possibly bad) given starting configuration. Section 4 focuses on sorting. Section 5 introduces two insertion strategies that always guarantee that free space can be made connected. Moreover, we present strategies that achieve low (amortized or worst-case) cost per insertion. Some concluding thoughts are given in Section 6 .

\section{Preliminaries}

Motivated by our FPGA application, we model the problem as follows: Let $A$ be an array (e.g., a memory or FPGA columns) that consists of $|A|$ cells. A module $M_{i}$ of size $m_{i}$ occupies a subarray of size $m_{i}$ in $A$ (i.e., $m_{i}$ consecutive cells). We call a subarray of maximal size where no module is placed a free space. The $i$ th free space (numbered from left to right) is denoted by $F_{i}$ and its size by $f_{i}$.

A module located in a subarray, $A_{s}$, can be moved to another subarray, $A_{t}$, if $A_{t}$ is of the same size as $A_{s}$ and all cells in $A_{t}$ are empty (particularly, both subarrays do not have a cell in common). Moves are distinguished into shifts and flips: If there is at least one module located between $A_{s}$ and $A_{t}$ we call the move a flip, otherwise a shift; see Fig. 1. Following the two approaches mentioned in the introduction, we are mainly interested in the following problems.

Offline Defragmentation: We start with a given configuration of modules in an array $A$ and look for a sequence of moves such that there is a free space of maximum size. We state the problem formally:

Given: An array $A$, and a set of modules, $M_{1}, M_{2}, \ldots, M_{n}$, placed in $A$.

Task: Move the modules such that there is a free space of maximum size.

Online Storage Allocation: This problem arises from inserting a sequence of modules, $M_{1}, M_{2}, \ldots, M_{n}$, which arrive in an online fashion, the next module arrives after the previous one has been inserted. After insertion, a module stays for some period of time in the array before it is removed; the duration is not known when placing an object. If an arriving module cannot be placed (because there is no sufficient connected free space), it has to wait until the array is compacted or other modules are removed. The modules in the array can be moved as described above to create free space for further insertions.

Our goals are twofold: On the one hand we want to minimize the makespan (i.e., the time until the last module is removed from the array) and, on the other hand, we want to minimize the costs for the moves. Moves are charged using a function, $c\left(m_{i}\right)$, which is linear in $m_{i}$. For example, we can simply count the number of moves using $c_{1}\left(m_{i}\right):=1$, or we count the moved mass (i.e., we sum up the sizes of the moved modules) with $c_{2}\left(m_{i}\right):=m_{i}$. Formally:

Given: An empty array, $A$, a sequence of modules, $M_{1}, M_{2}, \ldots, M_{n}$, arriving one after the other.

Task: Place the modules in $A$ such that (1) the makespan and (2) the total costs for all moves performed during the insertions is minimized. 


\section{Offline Defragmentation}

In this section, we assume that we are given an array that already contains $n$ modules. Our task is to compact the array; that is, move the modules such that we end up with one connected free space. Note that a practical motivation in the context of dynamic FPGA reconfiguation as well as some heuristics were already given in our paper [1]. As they lay the basis of some of the ideas in the following sections and for the sake of completeness, we briefly cite and sketch the corresponding complexity results.

Theorem 1 Rearranging an array with modules $M_{1}, \ldots, M_{n}$ and free spaces $F_{1}, \ldots, F_{k}$ such that there is a free space of maximum size is strongly NPcomplete. Moreover, there is no deterministic polynomial-time approximation algorithm within any polynomial approximation factor (unless $P=N P$ ).

The proof is based on a reduction of 3-PARTITION, see Figure 2. The sizes of the first $3 k$ modules correspond to the input of a 3-PARTITION instance, the size of the free spaces, $B$, is the bound from the 3-PARTITION instance. We can achieve a free space of maximum size, if and only if we can move the first $3 k$ modules to the free spaces, which corresponds to a solution for the 3PARTITION instance. The inapproximability argument uses a chain of immobile modules of increasing size that can be moved once a 3-PARTITION has been found, see [1].

This hardness depends on a number of immobile modules, i.e., on relatively small free space. If we define for an array $A$ of length $|A|$ the density to be $\delta=\frac{1}{|A|} \sum_{i=1}^{n} m_{i}$, it is not hard to see that if

$$
\begin{gathered}
\delta \leq \frac{1}{2}-\frac{1}{2|A|} \cdot \max _{i=1, \ldots, n}\left\{m_{i}\right\} \quad \text { or } \\
\max _{i=1, \ldots, n}\left\{m_{i}\right\} \leq \max _{j=1, \ldots, k}\left\{f_{j}\right\} .
\end{gathered}
$$

is fulfilled, the total free space can always be connected with $2 n$ steps by Algorithm 1 which shifts all modules to the right in the first loop and all modules to the left in the second loop. Starting at the right and left end, respectively.

Theorem 2 Algorithm 1 connects the total free space with at most $2 n$ moves and uses $O(n)$ computing time.

In the following, we use the idea of Algorithm 1 for maintenance strategies that can accommodate any module for which there is sufficient total free space.

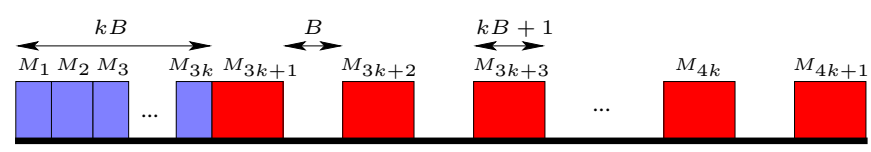

Fig. 2. Reducing 3-Partition to the MDP. 


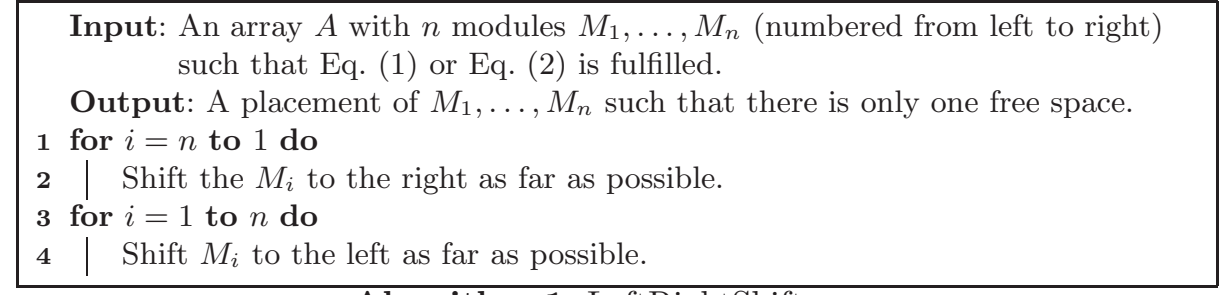

Algorithm 1: LeftRightShift

\section{Sorting}

In the next section, we present some strategies that are based on sorting the set of modules by their size. But more than that, sorting is always an important task. Thus, in this section we focus on the sorting problem for modules solely. Note that we cannot apply classical sorting algorithms such as Quicksort or Selection Sort, because they assume that every object is of the same size. We state an algorithm that is similar to Insertion Sort and show that it can be applied to our setting. It sorts $n$ modules in an array with $O\left(n^{2}\right)$ steps. Moreover we show that this is best possible up to a constant factor. More precisely, we deal with the following problem: Given an array, $A$, with modules $M_{1}, \ldots, M_{n}$ and free spaces $F_{1}, \ldots, F_{k}$. Sort the modules according to their size such that there is only one free space in $A$. It is necessary to be able to move every module. Therefore we assume in this section that Eq. (2) is fulfilled in the initial placement. Note that if Eq. (2) is not fulfilled, there are instances for which it is NP-hard to decide whether it can be sorted or not; this follows from a similar construction as in Section 3.

\subsection{Sorting $n$ modules with $O\left(n^{2}\right)$ steps}

To sort a given configuration, we first apply Algorithm 1, performing $O(n)$ moves. ${ }^{3}$ Afterwards, there is only one free space at the right end of $A$ and all modules are lying side by side in $A$. We number the modules in the resulting position from left to right from 1 to $n$. The algorithm maintains a list $I$ of unsorted modules. As long as $I$ is not empty, we proceed as follows: We flip the largest unsorted module, $M_{k}$, to the right end of the free space and shift all unsorted modules that were placed on the right side of $M_{k}$ to the left. Note that afterwards there is again only one free space in $A$.

Theorem 3 Let $A$ be an array with modules $M_{1}, \ldots, M_{n}$, free spaces $F_{1}, \ldots, F_{k}$, and let Eq. (2) be satisfied. Then Algorithm 2 sorts the array with $O\left(n^{2}\right)$ steps.

Proof. The while loop is executed at most $n$ times. In every iteration there is at most one flip and $n$ shifts. This yields an upper bound of $n^{2}$ on the total number of moves.

\footnotetext{
${ }^{3}$ A short proof of correctness for this procedure can be found in [1].
} 


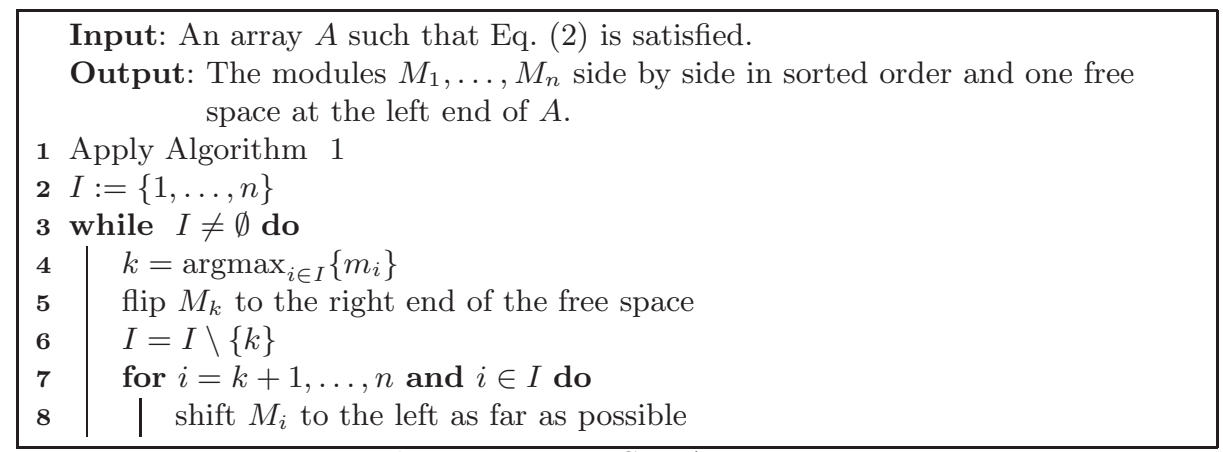

Algorithm 2: SortArray

For correctness, we prove the following invariant: At the end of an iteration of the while loop, all $M_{j}, j \notin I$, lie side by side at the right end of $A$ in increasing order (from left to right) and all $M_{j}, j \in I$, lie side by side at the left end of $A$. We call the first sequence of modules sorted and the other one non-sorted.

Now, assume that we are at the beginning of the $j$ th iteration of the while loop. Let $k$ be the index of the current maximum in $I$. By the induction hypothesis and by Eq. (2), the module $M_{k}$ can be flipped to the only free space. This step increases the number of sorted elements lying side by side at the right end of $A$. Since in every step the module of maximum is chosen, the increasing order in the sequence of sorted modules is preserved. Furthermore, this step creates a free space of size $m_{k}$ that divides the sequence of non-sorted modules into two (possible empty) subsequences. By the numbering of the modules, the left subsequence contains only indexes smaller than $k$. This ensures that in the second while loop only modules from the right subsequence are shifted. Again, since $M_{k}$ is chosen to be of maximum size all shifts are well defined. At the end of the iteration, the non-sorted modules lie side by side and so do the sorted ones.

\subsection{A Lower Bound of $\Omega\left(n^{2}\right)$}

We show that Algorithm 2 needs the minimum number of steps (up to a constant factor) to sort $n$ modules. In particular, we prove that any algorithm needs $\Omega\left(n^{2}\right)$ steps to sort the following example. The example consists of an even number of modules, $M_{1}, \ldots, M_{n}$, with size $m_{i}=k$ if $i$ is odd and $m_{i}=k+1$ if $i$ is even for a $k \geq 2$. There is only one free space of size $k+1$ in this initial placement at the left end of $A$, see Fig. 3 .

\begin{tabular}{|c|c|c|c|c|c|c|c|}
\hline & $M_{1}$ & $M_{2}$ & $M_{3}$ & $M_{4}$ & & $M_{n-}$ & $M_{n}$ \\
\hline$k+1$ & $k$ & $k+1$ & $k$ & $k+1$ & $\ldots$ & $k$ & $k+1$ \\
\hline
\end{tabular}

Fig. 3. Sorting an array is in $\Omega\left(n^{2}\right)$. 
Lemma 1 The following holds for any sequence of shifts and flips applied to the instance shown in Fig. 3:

(i) There are never two free spaces, each of size greater than or equal to $k$.

(ii) There might be more than one free space but there is always exactly one having either size $k$ or size $k+1$.

Proof. (i) is obvious because otherwise the sum of the sizes of the free spaces would exceed the total free space. (ii) follows because in the last step either a module of size $k$ or $k+1$ was moved leaving a free of size $k$ or $k+1$, resp.

Lemma 2 Let $A L G$ be an algorithm that uses a minimum number of steps to sort the above instance. Then the following holds:

(i) There is never more than one free space in $A$.

(ii) A module of size $k$ will only be shifted (and never be flipped).

Proof. Consider a step that created more than one free space. This is possible only if a module, $M_{i}$, of size $k$ was moved (i.e., there is one free space of size $k$ ). By Lemma 1, all other free spaces have sizes less than $k$. Thus, only a module, $M_{j}$, of size $k$ can be moved in the next step. Since we care only about the order of the sizes of the modules not about their numbering the same arrangement can be obtained by moving $M_{j}$ to the current place of $M_{i}$ and omitting the flip of $M_{i}$ (i.e., the number of steps in $A L G$ can be decreased); a contradiction.

From (i) we know that there is always one free space of size $k+1$ during the execution of $A L G$. Flipping a small module to this free space creates at least two free spaces. Hence, a small module will only be shifted.

Theorem 4 Any algorithm that sorts the modules in the example from Fig. 3 needs at least $\Omega\left(n^{2}\right)$ steps.

Proof. Let $A L G$ be an algorithm that needs the minimum number of steps. W.l.o.g. we assume that at the end the large modules are on the left side of the small ones. We consider the array in its initial configuration and, in particular, a module, $M_{i}$, of size $k$. There are $\frac{i-1}{2}$ small modules, the same number of large modules and one free space of size $k+1$ to the left of $M_{i}$. Because small modules are only shifted in $A L G$ the number of small modules on the left side of $M_{i}$ will not change but the number of large ones will finally increase to $\frac{n}{2}$. Since a shift moves $M_{i}$ at most a distance of $k+1$ to the right, $M_{i}$ has to be shifted at least once for every large module that is moved to $M_{i}$ 's left. Taking the free space into account this implies that $M_{i}$ has to be shifted at least $\frac{n}{2}-\left(\frac{i-1}{2}+1\right)$ times, for any odd $i$ between 1 and $n$. Hence, for $i=2 j-1$ we get a lower bound of $\sum_{j=1}^{\frac{n}{2}} \frac{n}{2}-j=\frac{1}{8} n^{2}-\frac{1}{4} n$ on the number of shifts in $A L G$. Additionally, every large module has to be flipped at least once, because it has a small one to its left in the initial configuration. This gives a lower bound of $\frac{1}{8} n^{2}-\frac{1}{4} n+\frac{1}{2} n=\frac{1}{8} n^{2}+\frac{1}{4} n$ on the total number of steps in $A L G$ and therefore a lower bound on the number of steps for any algorithm. 


\section{Strategies for Online Storage Allocation}

Now, we consider the online storage allocation problem, i.e., we assume that we have the opportunity to start with an empty array and are able to control the placement of modules. We consider strategies that handle the insertion and deletion of a sequence of modules. AlwaysSorted achieves an optimal makespan, possibly at the expense of requiring up to $O\left(n^{2}\right)$ moves per insertion; the algorithm ClassSort that is designed to require very few moves, but at the cost of larger makespan. Additionally, we present a simple local heuristic, LocalShift.

AlwaysSorted. This algorithm inserts the modules such that they are sorted according to their size; that is, the module sizes decrease from left to right. Note that the sorted order ensures that if a module, $M_{i}$, is removed from the array all modules lying on the right side of $M_{i}$ (these are at most as large as $M_{i}$ ) can be shifted $m_{i}$ units to the left. Now the algorithm works as follows: Before a module, $M_{j}$, is inserted, we shift all modules to the left as far as possible starting at the left side of the array. Next we search for the position that $M_{j}$ should have in the array to keep the sorted order. We shift all modules lying on the right side of the position $m_{j}$ units to the right if possible; after that $M_{j}$ is inserted.

Theorem 5 AlwaysSorted achieves the optimal makespan. The algorithm performs $O(n)$ moves per insertion in the worst case.

Proof. All modules are shifted to the left as far as possible before the next module is inserted. After that, there is only one free space at the right side of $A$. If this free space is at least as large as the next module, the insertion is performed, meaning that a module has to wait if and only if the total free space is smaller than the module size; no algorithm can do better.

DelayedSort. The idea is to reduce the number of moves by delaying the sorting until it is really necessary: We maintain a large free space on the left or the right side (alternatingly). First, we check if we can insert the current module $M_{i}$, i.e., if $m_{i} \leq \sum f_{j}$. Now, if we can insert $M_{i}$ maintaining $\max m_{i} \leq \max f_{j}$ we insert $M_{i}$ using First-Fit. Otherwise, we check if $M_{i}$ can be inserted-maintaining the above condition - after compacting the array using by shifting all modules to the side where we currently keep the large free space, beginning with the module next to the free space. If maintaining the condition is not possible, we sort the array using Alg. 2 and insert the module into the single free space left after sorting. Note that this strategy also achieves the optimal makespan.

ClassSort. For this strategy we assume that the size of the largest module at most half the size of the array. We round the size of a module, $M_{i}$, to the next larger power of 2; we denote the rounded size by $m_{i}^{\prime}$.

We organize the array in $a=\left\lceil\lg \frac{|A|}{2}\right\rceil$ classes, $C_{0}, C_{1}, \ldots, C_{a}$. Class $C_{i}$ has level $i$ and stores modules of rounded size $2^{i}$. In addition, each class reserves 0 , 1 , or 2 (initially 1) buffers for further insertions. A buffer of level $i$ is a free space of size $2^{i}$. We store the classes sorted by their level in decreasing order. 
The numbers of buffers in the classes provide a sequence, $S=s_{a}, \ldots, s_{0}$, with $s_{i} \in\{0,1,2\}$. We consider this sequence as a redundant binary number; see Brodal [14]. Redundant binary numbers use a third digit to allow additional freedom in the representation of the counter value. More precisely, the binary number $d_{\ell} d_{\ell-1} \ldots d_{0}$ with $d_{i} \in\{0,1,2\}$ represents the value $\sum_{i=0}^{\ell} d_{i} 2^{i}$. Thus, for example, $4_{10}$ can be represented as $100_{2}, 012_{2}$, or $020_{2}$. A redundant binary number is regular, if and only if between two 2's there is one 0 , and between two 0 's there is one 2 . The advantage of regular redundant binary numbers is that we can add or subtract values of $2^{k}$ taking care of only $O(1)$ carries, while usual binary numbers with $\ell$ digits and $11 \ldots 1_{2}+1_{2}=100 \ldots 0_{2}$ cause $\ell$ carries.

Inserting and deleting modules benefits from this advantage: The reorganization of the array on insertions and deletions corresponds to subtracting or adding, respectively, an appropriate value $2^{k}$ to the regular redundant binary numbers that represents the sequence $S$. In details: If a module, $M_{j}$, with $m_{j}^{\prime}=2^{i}$ arrives, we store the module in a buffer of the corresponding class $C_{i} \cdot{ }^{4}$ If there is no buffer available in $C_{i}$, we have a carry in the counter value; that is, we split one buffer of level $i+1$ to two buffers of level $i$; corresponding, for example, to a transition of $\ldots 20 \ldots$ to $\ldots 12 \ldots$ in the counter. Then, we subtract $2^{i}$ and get $\ldots 11 \ldots$ Now, the counter may be irregular; thus, we have to change another digit. The regularity guarantees that we change only $O(1)$ digits [14]. Similarly, deleting a module with $m_{j}^{\prime}=2^{i}$ corresponds to adding $2^{i}$ to $S$.

Theorem 6 ClassSort performs $O(1)$ moves per insertion or deletion in the worst case. Let $\hat{m}$ be the size of the largest module in the array, $c$ a linear function and $c\left(m_{i}\right)$ the cost of moving a module of size $m_{i}$. Then the amortized cost for inserting or deleting a module of size $m_{i}$ is $O\left(m_{i} \lg \hat{m}\right)$.

Proof. The number of moves is clear. Now, observe a class, $C_{i}$. A module of size $2^{i}$ is moved, if the counter of the next smaller class, $C_{i-1}$, switches from 0 to 2 (for the insertion case). Because of the regular structure of the counter, we have to insert at least modules with a total weight of $2^{i-1}$ before we have to move a module of size $2^{i}$ again. We charge the cost for this move to theses modules. On the other hand, we charge every module at most once for every class. As we have $\lg \hat{m}$ ) classes, the stated bound follows. The same argument holds for the case of deletion. Note that we move modules only, if the free space inside a class is not located on the right side of the class (for insertion) or on the left side (for deletion). Thus, alternatingly inserting and deleting a module of the same size does not result in a large number of moves, because we just imaginarily split and merge free spaces.

LocalShift. We define the distance between two blocks (modules or free spaces) as the number of blocks that lie between these two blocks. For a free space $F_{i}$ we call the set of blocks that are at most at a distance $k \in \mathbb{N}$ from $F_{i}$ the $k$-neighborhood of $F_{i}$. The algorithm LocalShift works as follows: If possible

\footnotetext{
${ }^{4}$ Initially, the array is empty. Thus, we create the classes $C_{1}, \ldots, C_{i}$ if they do not already exist, reserving one free space of size $2^{k}$ for every class $C_{k}$.
} 

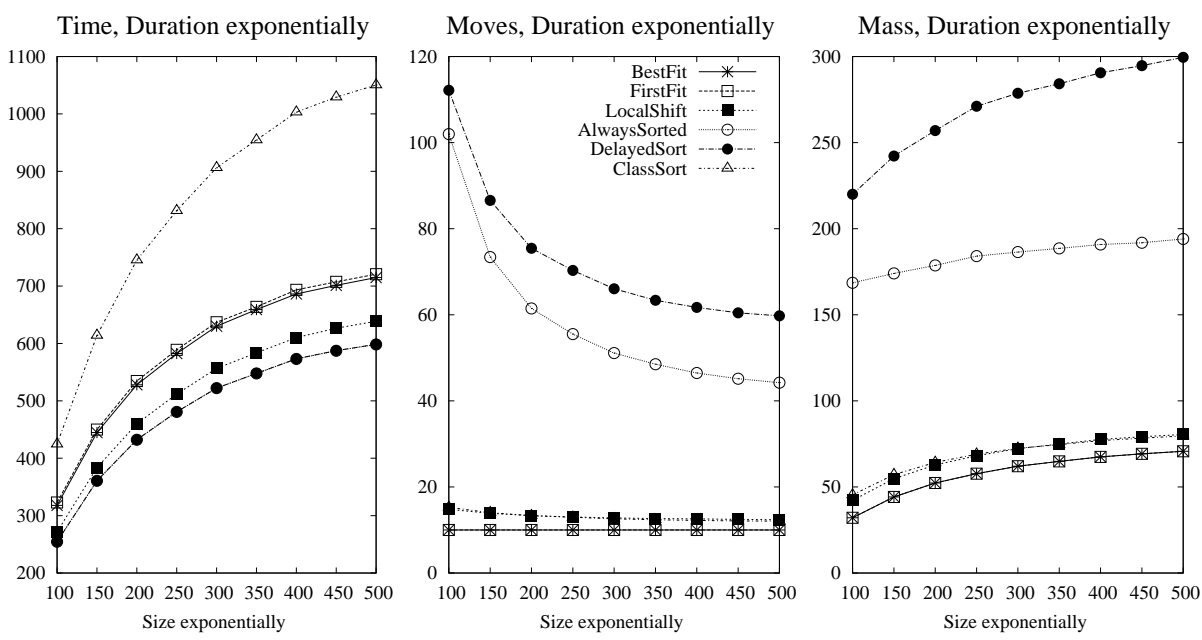

Fig. 4. Experiments with exponential distribution for size and duration.

we use BestFit to insert the next module $M_{j}$. Otherwise, we look at the $k$ neighborhood of any free space (from left to right). If shifting the modules from the $k$-neighborhood, lying on the left side of $F_{i}$, to the left as far as possible (starting a the left side) and the modules lying on the right side to the right as far as possible (starting at the right side) would create a free space that is at least as large as $M_{j}$ we actually perform these shifts and insert $M_{j}$. If no such free space can be created, $M_{j}$ has to wait until at least one modules is removed from the array. This algorithm performs at most $2 k$ moves per insertion.

\section{Comparison and Conclusion}

To test our strategies, we generated a number of random input sequences and analyzed the performance of our strategies as well as the simple FirstFit and BestFit approaches in an array of size $2^{10}$. A sequence consists of 100,000 modules, each module has a randomly chosen size and duration time. For each sequence, size and time are shuffled using several probability distributions. We analyzed three objectives: the time to complete the whole sequence (the makespan), the number of moved modules $\left(c\left(m_{i}\right)=1\right)$ and the moved mass $\left(c\left(m_{i}\right)=m_{i}\right)$. Our experiments (see Fig. 4 for an example) showed that LocalShift performs very well, as it constitutes a compromise between a moderate number moves and a low makespan. Both makespan and moves turn out to be nearly optimal.

The more complex strategy ClassSort performed only slightly worse than LocalShift concerning moves, but disappoints in its resulting makespan. In contrast, both types of sorting-related strategies have - of course - a good makespan, but need a lot of moves. Unsurprisingly, FirstFit and BestFit need the fewest moves (as they perform moves only on inserting a module, but never move a previously 
placed module). Their makespan turned out to be clearly better than ClassSort, but worse than LocalShift and the sorting strategies.

A comparison of the sorting strategies, AlwaysSorted and DelayedSort, showed that delaying the sorting of the array until it is really necessary pays off for the number of moves, but not if we count the moved mass, this is because the shift from maintaining one large free space to sorting (caused by not enough free space to accompany the largest item) results in a sequence with several moves of the heaviest items, which is not the case for AlwaysSorted.

We have introduced the systematic study of dynamic storage allocation for contiguous objects. There are still a number of open questions, such as the worst-case number of moves required to achieve connected free space or cheaper certificates for guaranteeing that connected free space can be achieved.

\section{References}

[1] Fekete, S.P., Kamphans, T., Schweer, N., Tessars, C., van der Veen, J.C., Angermeier, J., Koch, D., Teich, J.: No-break dynamic defragmentation of reconfigurable devices. In: Proc. Internat. Conf. Field Program. Logic Appl. (FPL 08). (2008)

[2] Knuth, D.E.: The Art of Computer Programming: Fundamental Algorithms. Third edn. Volume 1. Addison-Wesley (1997)

[3] Luby, M.G., Naor, J., Orda, A.: Tight bounds for dynamic storage allocation. SIAM Journal on Discrete Math. 9 (1996) 155-166

[4] Naor, J., Orda, A., Y.Petruschka: Dynamic storage allocation with known durations. Discrete Applied Mathematics 3 (2000) 203-213

[5] Coffman, E.G., Garey, M.R., Johnson, D.S.: Approximation algorithms for bin packing: A survey. In Hochbaum, D.S., ed.: Approximation Algorithms for NPHard Problems. PWS Publishing Company, Boston, MA (1996) 46-93

[6] Knuth, D.E.: The Art of Computer Programming: Sorting and Searching. Third edn. Volume 3. Addison-Wesley (1997)

[7] Willard, D.E.: A density control algorithm for doing insertions and deletions in a sequentially ordered file in good worst-case time. Information and Computation 97 (1992) 150-204

[8] Bender, M.A., Demaine, E.D., Farach-Colton, M.: Cache-oblivious B-trees. SIAM J. Comput 35 (2005) 341-358

[9] Bender, M.A., Hu, H.: An adaptive packed-memory array. Transactions on Database Systems 32 (2007) Special Issue on PODS '06.

[10] Bender, M.A., Farach-Colton, M., Mosteiro, M.A.: Insertion sort is $\mathrm{O}(\mathrm{n} \log \mathrm{n})$. Theory of Computing Systems 39 (2006) 391-397 Special Issue on FUN '04.

[11] Gue, K.R., Kim, B.S.: Puzzle-based storage systems. TR, Auburn University (2006)

[12] Kenyon, C., Remila, E.: Approximate strip packing. In: Proc. 37th Annu. IEEE Sympos. Found. Comput. Sci. (1996) 31-36

[13] Augustine, J., Banerjee, S., Irani, S.: Strip packing with precedence constraints and strip packing with release times. In: Proc. 18th Annu. ACM Sympos. Parallel Algor. Architect. (2006) 180-189

[14] Brodal, G.S.: Worst-case efficient priority queues. In: Proceedings of the Seventh Annual ACM-SIAM Symposium on Discrete Algorithms (SODA 96), Atlanta, Georgia (1996) 52-58 